OPEN

SUBJECT AREAS:

OPTICAL MANIPULATION AND TWEEZERS

NANOWIRES

Received

3 December 2013

Accepted

20 January 2014

Published

5 February 2014

Correspondence and requests for materials should be addressed to B.J.L. (stslbj@outlook. com)

\section{Controllable orientation of single silver nanowire using two fiber probes}

\author{
Xiaohao Xu, Chang Cheng, Hongbao Xin, Hongxiang Lei \& Baojun Li
}

State Key Laboratory of Optoelectronic Materials and Technologies, School of Physics and Engineering, Sun Yat-Sen University, Guangzhou 510275, China.

We report a strategy for realizing precise orientation of single silver nanowire using two fiber probes. By launching a laser of $980 \mathrm{~nm}$ wavelength into the two fibers, single silver nanowire with a diameter of $600 \mathrm{~nm}$ and a length of $6.5 \mu \mathrm{m}$ suspended in water was trapped and rotated by optical torque resulting from its interaction with optical fields outputted from the fiber probes. Angular orientation of the nanowire was controlled by varying the relative distance between the two fiber probes. The angular stiffness, which refers to the stability of orientation, was estimated to be on the order of $10^{-19} \mathrm{~J} / \mathrm{rad}^{2} \cdot \mathrm{mW}$. The experiments were interpreted by theoretical analysis.

$\mathrm{N}$ oble metal nanowires are currently known as a promising element in nanophotonic integration because of fascinating optical properties caused by localized surface plasmon resonances for concentrating and guiding light in sub-wavelength volumes ${ }^{1,2}$. Although chemical nanowire synthesis techniques are matured, application of metal nanowires in nanophotonic systems is greatly restricted. One of the most important challenges is how to assemble metal nanowires into multi-functional photonic systems. To "pick-and-place" onedimentional nanostructures (e.g. metal nanowires, carbon nanotubes, semiconductor nanowires, etc.) in different assemblies so that to assemble nanophotonic devices such as plasmon routers and optical antennas, both high spatial precision and angular precision are required ${ }^{3-5}$. In addition, to characterize plasmonic nanowires, ability to accurately control the angle and orientation of one-dimentional nanostructures is also desired because the plasmonic responses are sensitive to angular orientation ${ }^{6,7}$. Therefore, orienting metal nanowire with high angular precision is of crucial important for serving as functional elements in nanophotonic systems and characterization ${ }^{8}$. Particularly, direct orientation of single nanowire in liquid medium is even more difficult because the nanowire will be affected by the Brownian motion and the environmental fluctuation.

Optical forces provide a powerful approach to manipulate objects ${ }^{9-11}$. The ability of light to rotate and orient objects by optical torque makes it more versatile to construct specific semiconductor nanowires, which may function as active photonic devices ${ }^{10}$. Traditionally, optical torque for rotating objects can be generated by transferring spin or orbital angular momentum of laser beam to the objects ${ }^{12,13}$. Based on the reported mechanisms $^{12-15}$, continuous rotation of objects has been realized by utilizing circular polarization or Laguerre-Gaussian beams. Using the polarization dependent optical forces ${ }^{6,16}$, orientation of silver nanowires has also been realized. To conveniently orient objects with high precision, a simple and low-cost method for orienting single silver nanowire can facilitate the applications of silver nanowire in nanophotonic systems. Fortunately, recent report indicates that fiber probes are powerful in manipulating microscopic particles ${ }^{17}$, which motives us to try to manipulate silver nanowire precisely using fiber probes without the need of complicated optical components such as high-numerical-aperture objective, wave plates, and spatial light modulators. Therefore, in this work, we report a controllable orientation of single silver nanowire through optical torque. Optical torque is caused by radiation pressures which arises from the optical fields outputted from the two fiber probes and directly exerted on the silver nanowire.

\section{Results}

Optical orientation of single silver nanowire. Figure 1a schematically shows the experimental setup. Silver nanowires were synthesized via a polyol process ${ }^{18}$ with diameters ranging from 100 to $700 \mathrm{~nm}$ and lengths ranging from 2 to $12 \mu \mathrm{m}$ (see Fig. S1 in Supplementary Information for the SEM image and distribution). To orient single silver nanowire, a laser of $980 \mathrm{~nm}$ wavelength was coupled into fiber probe 1 (FP1) and fiber probe 2 (FP2) (see Methods for fabrication) by a Y-branch coupler (1:1 splitting ratio). The two FPs are fixed by two tunable microstages (see Methods for description) in an opposite direction, respectively, and the tips of the FPs 

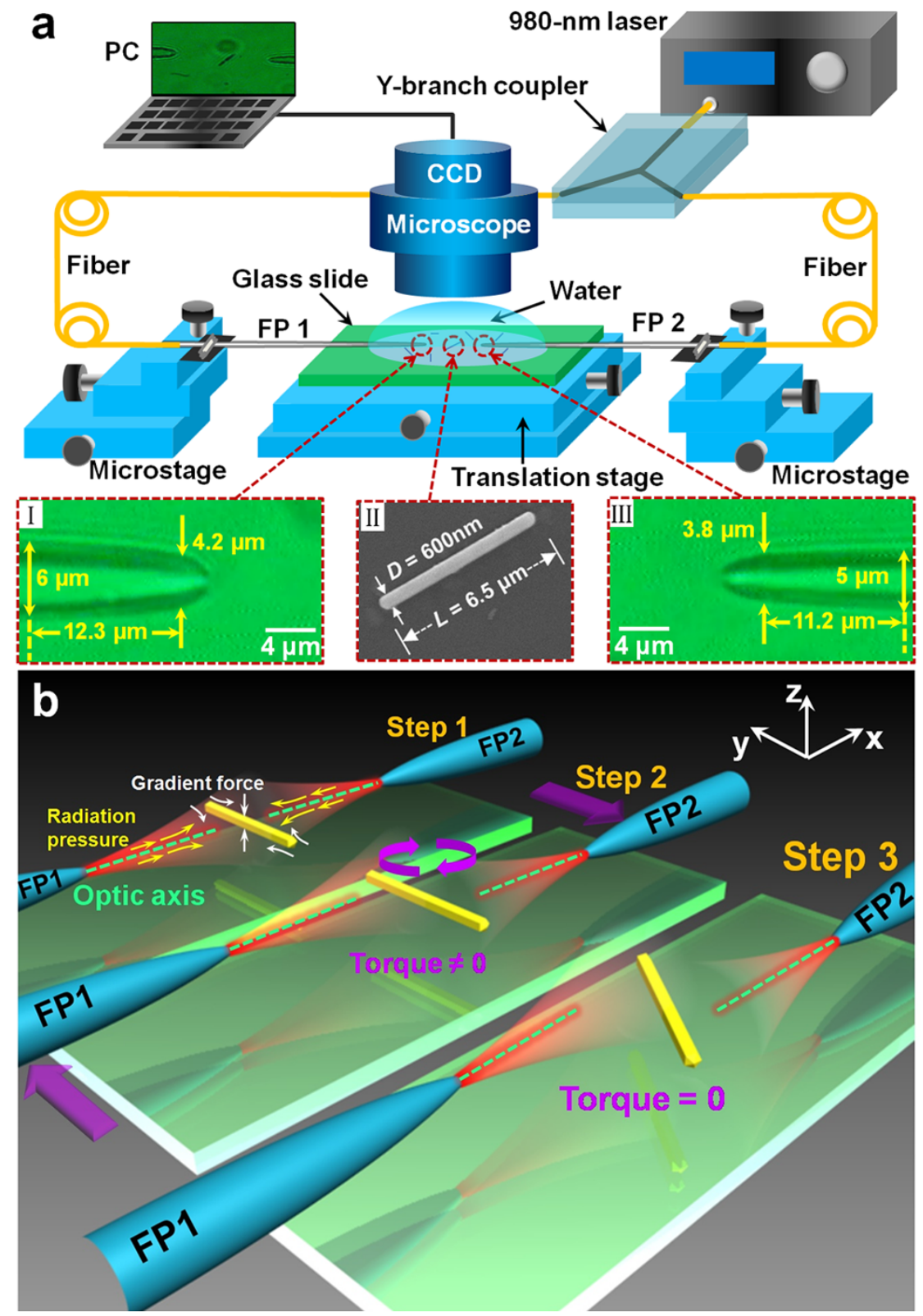

Figure 1 Experimental setup and manipulation process. (a) Schematic of experimental setup. Insets I and III indicate optical microscope images of FP1 and FP2, respectively. Inset II shows the scanning electron micrograph of a single nanowire. (b) Schematic manipulation process of a single silver nanowire using two FPs. Step 1 shows a single silver nanowire is trapped by two counter-propagating beams. By moving the two FPs along opposite directions, the nanowire is rotated by the optical torque (step 2). Once the FPs stop moving, the nanowire will be stationary with a new orientation (step 3 ).

are immersed in the suspension of silver nanowires (see Methods for preparation). The diameters of the FP1 and FP2 are decreased from 6 to $4.2 \mu \mathrm{m}$ in $12.3 \mu \mathrm{m}$ long and from 5 to $3.8 \mu \mathrm{m}$ in $11.2 \mu \mathrm{m}$ long, respectively, as shown in the insets I and III of Fig. 1a. Inset II of Fig. 1a shows the image of a typical silver nanowire with a length of $6.5 \mu \mathrm{m}$ and a diameter of $600 \mathrm{~nm}$ used in following experiment (see Fig. S2 in Supplementary Information for the scattering spectrum obtained by dark field illumination). To understand the orientation mechanism, a schematic for manipulating silver nanowire is illustrated in Fig. 1b and described as follows: Step 1, by moving the FP1 and FP2 to make sure that the nanowire is between the two probes and then launching laser beams into the FP1 and FP2 to trap the silver nanowire by two counter-propagating beams. Step 2, by moving the FP1 and FP2 oppositely along the $y$ direction to rotate the nanowire through a non-zero optical torque which is generated by the asymmetric distribution of radiation pressures.
Once the moving of the FPs is stopped, the rotation of the nanowire will also be stopped with an orientation where the two competing radiation pressures are offset (i.e. optical torque equals zero) as depicted in Step 3. By altering the distance between the FP1 and FP2 in $y$ direction, orientation of the nanowire can be controlled.

Figure 2 shows the consecutive images for rotation of the silver nanowire with a length of $6.5 \mu \mathrm{m}$ and a diameter of $600 \mathrm{~nm}$ by altering the distance $(s)$ between the FP1 and FP2 in $y$ direction. Right panels show corresponding angular orientation $(\theta)$ of the nanowire. The distance between the two FP tips in $x$ direction is fixed at $d=23 \mu \mathrm{m}$. It can be seen that, at the beginning $(s=$ $4.0 \mu \mathrm{m})$, the wire was trapped by the radiation pressures with $\theta=$ $82^{\circ}$ (Fig. 2a). By moving the FP1 in $+y$ direction and the FP2 in $-y$ direction simultaneously, the wire was rotated clockwise due to the non-zero optical torque generated by the change of radiation pressures. Figure $2 \mathrm{a}-\mathrm{e}$ shows the angular orientation $\theta$ of the wire 


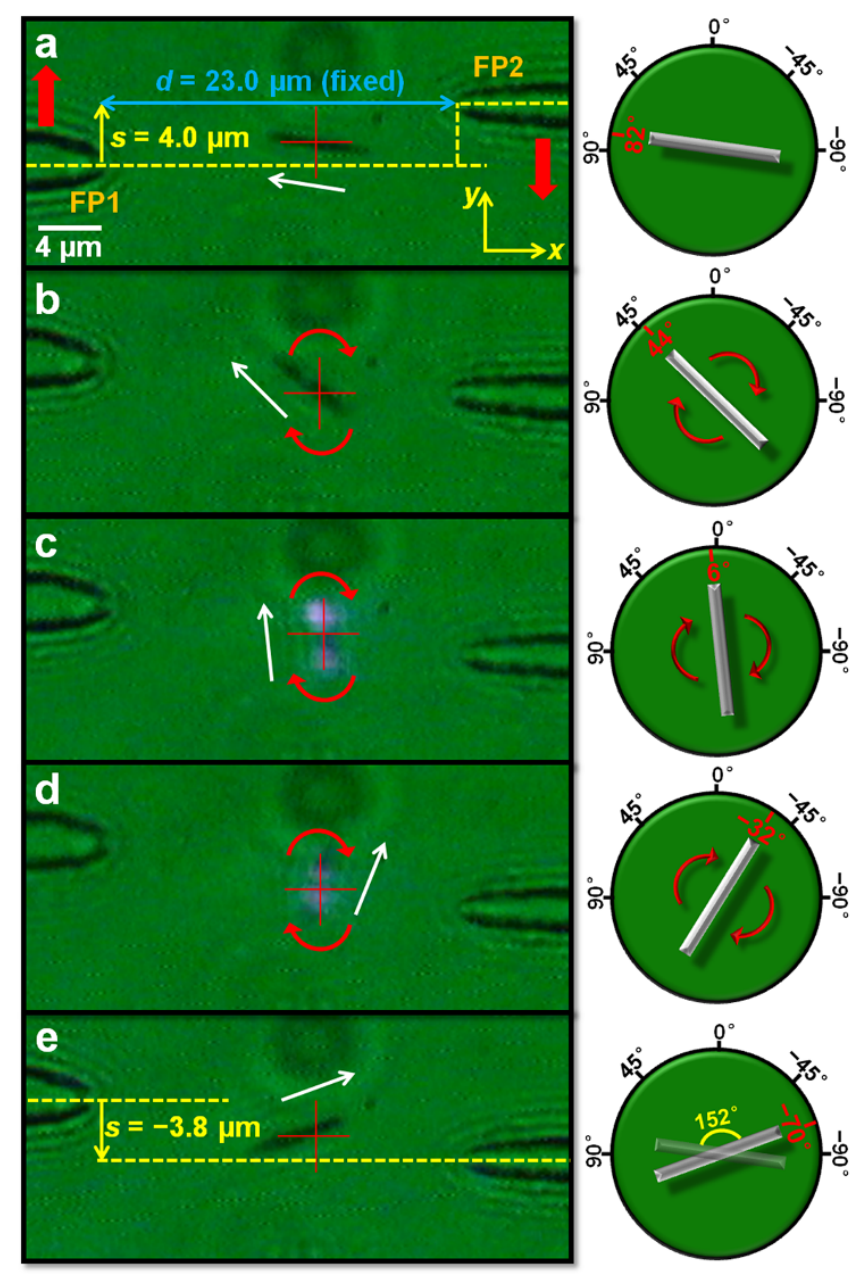

Figure $2 \mid$ CCD captured microscope images for rotation process of the silver nanowire with length of $6.5 \mu \mathrm{m}$ and diameter of $600 \mathrm{~nm}$ using two fiber probes with an input optical power of $18 \mathrm{~mW}$ in each fiber probe. (a-e) Rotation of the wire in response to a decreasing difference $s$ between the FP1 and FP2 in $y$ direction. The crossed red symbol is used as a reference position, the white arrow labels the orientation of the nanowire, and the dashed yellow line indicates the axis of each fiber. Right panels show corresponding angular orientation of the nanowire for (a-e).

continuously changed from $82^{\circ}$ to $-70^{\circ}$ with an interval of $-38^{\circ}$. It can be seen that, the nanowire was rotated $152^{\circ}$ while the $s$ was changed from 4.0 to $-3.8 \mu \mathrm{m}$. It should be pointed out that the nanowire can also be rotated counterclockwise by inverse process because of the symmetry. In our experiment, we found that when $|s|$ $>6.0 \mu \mathrm{m}$, the optical torque is too weak to rotate the nanowire, which in turn limits the range of variation of $\theta$. Fortunately, the limitation can be overcome by increasing the output power in each fiber or decreasing the distance $(d)$ between the two FP tips in $x$ direction. It should be noted that an obvious scattering light was occurred in Fig. 2c,d. The reason is that the nanowire is almost perpendicular to the FPs, which caused the strongest scattering light recorded by the CCD.

To know the orienting capability, the dependence of angular orientation at equilibrium state (defined as $\theta_{0}$ ) versus $s$ was investigated. Figure 3a1-e1 shows that, when $s$ is changed from -3.2 to $-1.2,0.0$, 0.8 , and $2.3 \mu \mathrm{m}, \theta_{0}$ is changed from $-64^{\circ}$ to $-32^{\circ}, 0^{\circ}, 32^{\circ}$, and $64^{\circ}$, respectively. Since the sizes of the two FPs are slightly different, the midpoint (indicated by red dot in Fig. 3a1) of the trapped nanowire in $x$ direction is not in the middle line of two FPs. The deviation is $2.5 \mu \mathrm{m}$. It should be pointed out that the Brownian motion of the nanowire in water will result in a fluctuation of $\theta_{0}$ at equilibrium state. Figure 3a2-e2 shows the corresponding counted probability density of the angular displacement. The red line represents the normalized Gaussian curve: $P(\theta)=\left(2 \pi \sigma^{2}\right)^{-1 / 2} \cdot \exp \{-0.5[(\theta-\mu) /$ $\left.\sigma]^{2}\right\}$, where $\sigma$ and $\mu$ are fitting parameters. It can be seen that, the standard deviation $\sigma$, which is positively related to the angular fluctuations, is $2^{\circ} \sim 3^{\circ}$. Moreover, for a larger absolute value $s, \sigma$ is larger. Additionally, from the distributions of angular orientation, the stability of orientation can also be quantitatively estimated by calculating the stiffness coefficient $\kappa$, which is defined as $\kappa=k_{\mathrm{B}} \cdot T /\left\langle\theta_{0}{ }^{2}\right\rangle$ (Ref. 16), where $k_{\mathrm{B}}$ is the Boltzmann constant and $T$ is the temperature in Kelvin. The calculated stiffness coefficients are 0.8484, $1.1565,1.4588,1.0893$, and $1.0211 \times 10^{-17} \mathrm{~J} / \mathrm{rad}^{2}$ for $s=-3.2$, $-1.2,0.0,0.8$, and $2.3 \mu \mathrm{m}$, respectively. Figure $3 \mathrm{f}$ shows the measured $\theta_{0}$ as a function of $s$ (red line). It can be seen that $\theta_{0}$ increases with increasing $s$. Moreover, the increase rate of $\theta_{0}$ becomes slow with the increasing $|s|$, which is mainly attributed to that the change of radiation pressures with the distance becomes slow as the probe tips are away from the nanowire. The calculated stiffness coefficient $\kappa$ versus $s$ is also shown in Fig. $3 \mathrm{f}$ (blue line). The calculated average stiffness coefficient is $2.4 \times 10^{-19} \mathrm{~J} / \mathrm{rad}^{2} \cdot \mathrm{mW}$ for a laser power of $36 \mathrm{~mW}$. Moreover, there is a maximum $\kappa$ at $s=0$. This is because the optical torque, which constrains the fluctuations of angular orientation of the wire, is weaker when the probes are far away from the nanowire.

Theoretical simulation and calculation. To explain the orientation mechanism numerically, a theoretical model was built with a finite element simulation (COMSOL Multiphysics). The shape of the nanowire is approximated as a cylinder $(600 \mathrm{~nm}$ in diameter and $6.5 \mu \mathrm{m}$ in length). Each fiber probe is approximated as a cone with a parabolic body which was constructed by revolving parametric surfaces (see Table. S1 in Supplementary Information for geometric parameters). Benefiting from the tapered shape, the outputted laser beam is focused at the fiber tip (see Fig. S3). The focused narrow optical field distribution provides a radiation field along optic axis and a gradient field in $y$ - $z$ plane. Therefore, the nanowire can be trapped between the two counter-placed FPs. Figure S4 in Supplementary Information represents an overview of global geometrics. The input power of each FP is set to be $18 \mathrm{~mW}$. The refractive indices of FPs and water are set to be 1.45 and 1.33 , respectively. The refractive index of silver at $980 \mathrm{~nm}$ wavelength is $0.04+6.992 \mathrm{i}$ (Ref. 19). According to the above discussion, the center position of the nanowire is not in the middle line. So a deviation of $2.5 \mu \mathrm{m}$ is taken into account in the model. The distance from the midpoint of the nanowire to each FP is assumed to be same in $y$ direction. As an example, Figure $4 \mathrm{a}-\mathrm{c}$ shows the time averaged energy density distribution for $s=0$ and $\theta=0^{\circ}, 45^{\circ}$, and $-45^{\circ}$. It can be seen that, when $\theta=0^{\circ}$, the distribution of field around the nanowire is symmetric with respect to the middle transverse section of the nanowire. When $\theta=45^{\circ}$ or $-45^{\circ}$, the field is asymmetric. This asymmetric distribution will generate an optical torque exerted on the nanowire (see Fig. S5). From the simulations, it is easy to estimate the direction of rotation by calculating the optical torque exerted on the nanowire. To indicate the direction of optical torque, we define $+z$ direction as the positive direction. Thus the optical torque $M$ can be expressed as $^{9,20}$

$$
\boldsymbol{M}=\hat{\mathbf{z}} \cdot\left(\oiint \boldsymbol{r} \times\left\langle\mathrm{T}_{\mathrm{M}}\right\rangle \cdot \hat{\mathbf{n}} d A\right),
$$

where the integration is performed over a closed surface $A$ surrounding the nanowire, $\hat{\mathbf{n}}$ is the unit vector outward normal to the surface $A, r$ is the position vector with respect to the rotation axis, $\hat{\mathbf{z}}$ is the unit vector in $z$ direction, and $\left\langle\mathrm{T}_{\mathrm{M}}\right\rangle$ is the time averaged Maxwell stress tensor which can be expressed by 

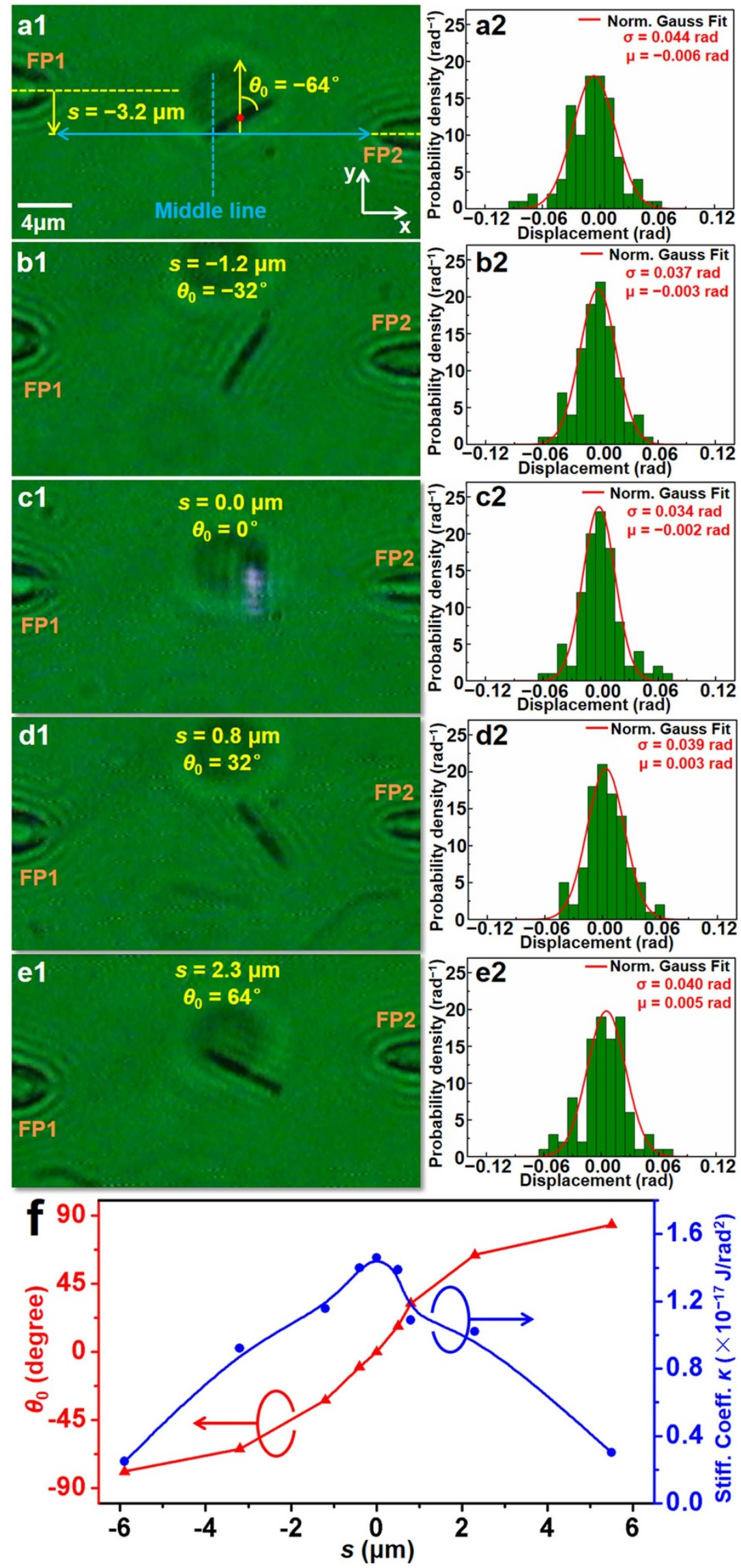

Figure $3 \mid$ Orienting capability and stiffness. (a1-e1) Microscopic images of the silver nanowire at equilibrium state with different $s$. (a2-e2) Probability density of the angular displacement. The angles are measured with an interval of $0.1 \mathrm{~s}$. (f) The angular orientation at equilibrium $\theta_{0}$ and stiffness coefficient of the nanowire as a function of $s$. 

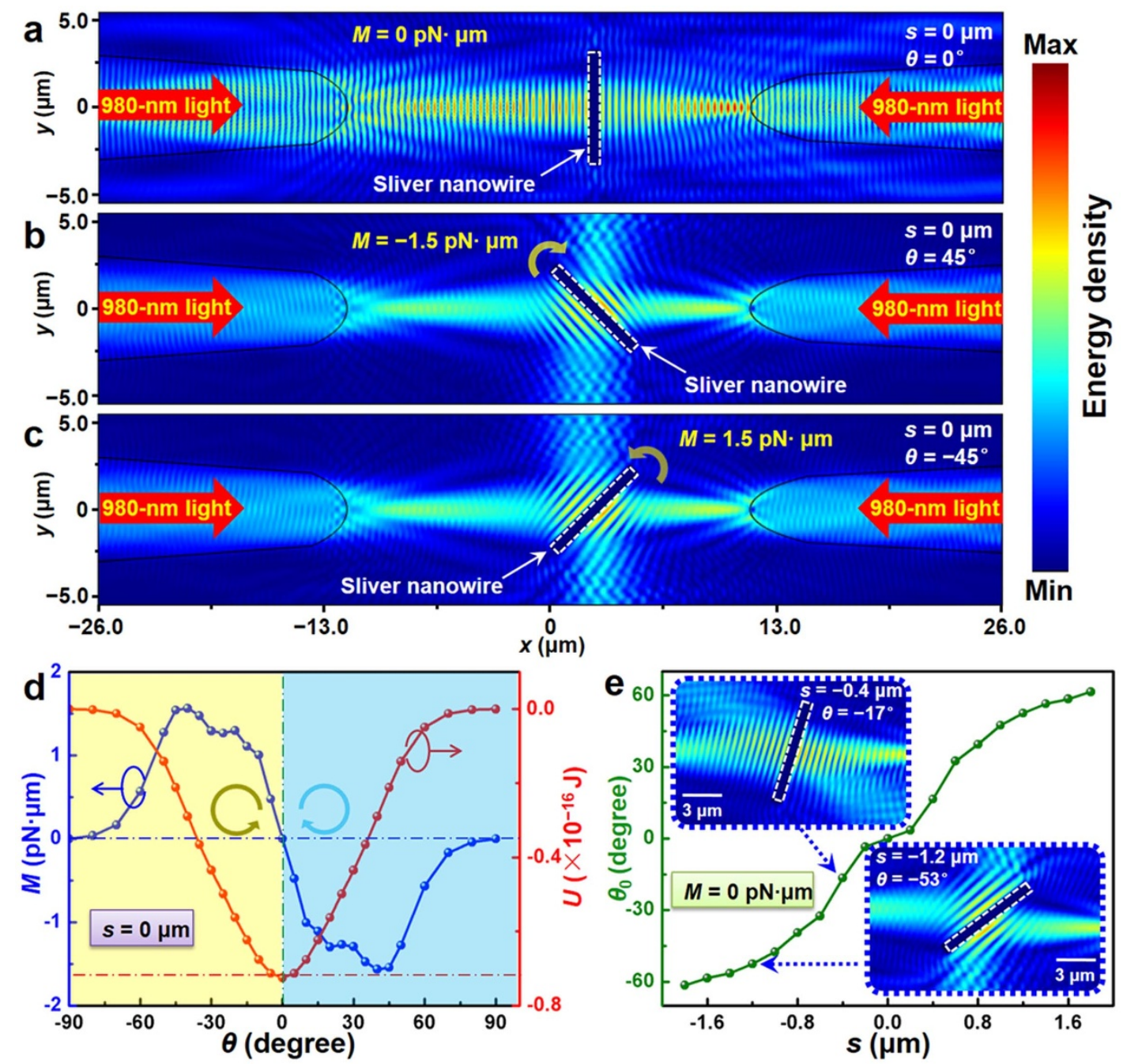

Figure $4 \mid$ Simulated time averaged energy density distribution and calculated optical torque. $(\mathrm{a}-\mathrm{c})$ Time averaged energy density distribution for $s=0$ and $\theta=0,45^{\circ},-45^{\circ}$. (d) Torque $M$ and rotational potential energy $U$ versus angular orientation $\theta$ for $s=0$. Yellow and blue regions indicate the anticlockwise and clockwise rotation, respectively. (e) Angular orientation at equilibrium state $\theta_{0}$ as a function of $s$. The two insets show the time averaged energy density distribution at equilibrium state for $s=-0.4$ and $-1.2 \mu \mathrm{m}$, respectively.

$$
\left\langle\mathrm{T}_{\mathrm{M}}\right\rangle=\frac{1}{2} \varepsilon\left(\mathbf{E} \otimes \mathbf{E}^{*}\right)+\frac{1}{2} \mu\left(\mathbf{H} \otimes \mathbf{H}^{*}\right)-\frac{1}{4}\left(\varepsilon|\mathbf{E}|^{2}+\mu|\mathbf{H}|^{2}\right) \mathbf{I},
$$

where $\otimes$ denotes dyadic product, $I$ is the unit dyadic, the superscript * indicates complex conjugation, $\varepsilon$ is the electric permittivity, and $\mu$ is the magnetic permeability of the surroundings. According to Eqs. (1) and (2), the calculated optical torque $M$ exerted on the nanowire are $0,-1.5$, and $1.5 \mathrm{pN} \cdot \mu \mathrm{m}$, which induce the nanowire to be halted, rotated clockwise, and rotated anticlockwise, respectively.

To further investigate the influence of the $s$ on the angular orientation at equilibrium state $\theta_{0}$, a series of simulations were carried out. For a particular $s$, the corresponding $\theta_{0}$ can be obtained by analyzing the relation between the calculated $M$ and the angular orientation $\theta$. Figure $4 \mathrm{~d}$ shows the calculated $M$ versus angular orientation $\theta$ for $s=0$ (blue-dotted curve). It can be seen that when $0<\theta<90^{\circ}, M<0$. When $-90^{\circ}<\theta<0, M>0$. When $\theta=0$ and $90^{\circ}, M=0$. These indicate that the nanowire will be rotated towards $\theta=0^{\circ}$. The rotational potential energy $U$ is evaluated by integrating $M$ with respect to $\theta$ (red-dotted curve). It can be seen that $U$ reaches the minimum at $\theta=0$, which implies that this orientation is stable at equilibrium $\theta_{0}$ for $s=0$. Using the same methods, corresponding $\theta_{0}$ was obtained for $s$ from -1.8 to $1.8 \mu \mathrm{m}$ (Fig. $4 \mathrm{e}$ ). The results show that $\theta_{0}$ increases with increasing $s$. This is consistent with the former experimental results (Fig. 3f). The difference between the calculated and experimental results is mainly because the center of the nanowire is not always accurately equidistant from the two fiber probes in $y$ direction in experiment.

\section{Discussion}

The above experimental results show that controllable orientation of single silver nanowire can be realized using two FPs. To know the possibility of the method on different size nanowires or different composition nanowires, further simulations were carried out. Figure 5, as an example, shows calculated optical torque $M$ and corresponding rotational potential $U$ as a function of the angular orientation $\theta$ for the nanowires with several typical sizes at $s=0$. For comparison, the results of the nanowire with a size of diameter $(D) \times$ length $(L)=(0.6 \times 6.5) \mu \mathrm{m}$ used in this experiment was also added. It can be seen that, the trend and shape of $M$ for the nanowires with sizes of $(D \times L)=(0.4 \times 6.5),(0.8 \times 6.5),(0.6 \times 4)$, and $(0.6 \times$ 8) $\mu \mathrm{m}$ are similar to that of the nanowire with $(D \times L)=(0.6 \times 6.5)$ $\mu \mathrm{m}$ (Fig. 5a). This means that these nanowires can be rotated towards $\theta=0^{\circ}$. However, for the thinner nanowire with $(D \times L)=(0.2 \times$ 6.5) $\mu \mathrm{m}$ and the shorter nanowire with $(D \times L)=(0.6 \times 2) \mu \mathrm{m}$, the sign of $M$ will be changed at $|\theta|>77^{\circ}$ and $64^{\circ}$, respectively. It means that in this range of angle, the nanowire will be rotated towards $\theta=$ 

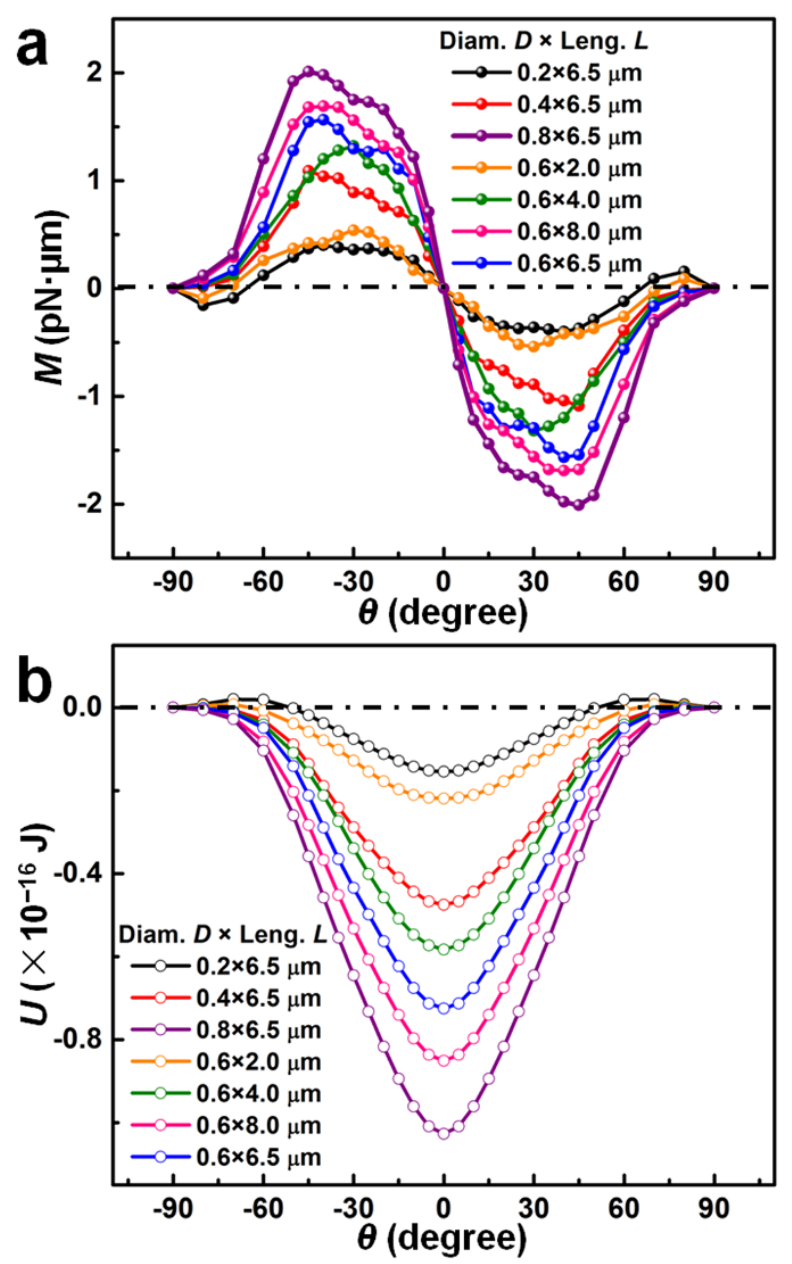

Figure $5 \mid$ Caculated optical torque $M$ and corresponding rotational potential $U$ for siver nanowires with different sizes for $s=0$. (a) $M$ versus $\theta$. (b) $U$ versus $\theta$.

$\pm 90^{\circ}$ (noting this two orientations are equivalent) rather than $\theta=$ $0^{\circ}$. Figure $5 \mathrm{~b}$ shows the corresponding rotational potential $U$ versus $\theta$. It can be seen that, $U$ reaches a minimum at $\theta=0^{\circ}$, indicating equilibrium state. The potential well depth $\Delta U_{\text {dep }}=\left|U_{\max }-U_{\min }\right|$, which is directly related to the average $M$, are calculated as $0.17,0.46$, $1.03,0.22,0.58,0.85$, and $0.72 \times 10^{-16} \mathrm{~J}$ for the silver nanowires with $(D \times L)=(0.2 \times 6.5),(0.4 \times 6.5),(0.8 \times 6.5),(0.6 \times 2),(0.6 \times 4)$, $(0.6 \times 8)$, and $(0.6 \times 6.5) \mu \mathrm{m}$, respectively. The results indicate that the $\Delta U_{\text {dep }}$ becomes deeper with increasing nanowire size, which is beneficial for orientation stability. In addition, there are another shallow potential wells in range $|\theta|>77^{\circ}$ and $64^{\circ}$ for nanowires with $(D \times L)=(0.2 \times 6.5)$ and $(0.6 \times 2) \mu \mathrm{m}$, respectively. The potential $U$ in these potential wells reaches the minimum at $\pm 90^{\circ}$ for both, which implies that this two silver nanowires can also be stable at $\theta= \pm 90^{\circ}$ with corresponding potential well depth $\Delta U_{\mathrm{dep}}=0.02 \times 10^{-16}$ and $0.01 \times 10^{-16} \mathrm{~J}$.

To know the capability in controlling different composition nanowires, such as dielectric compositions, the optical torque $M$ (plotted by solid symbols) and the corresponding rotational potential $U$ (plotted by hollow symbols) for both PMMA and $\mathrm{ZnO}$ nanowires with $(D$ $\times L)=(0.6 \times 6.5) \mu \mathrm{m}$ were calculated as shown in Fig. 6a. The refractive indices of the PMMA and $\mathrm{ZnO}$ are set as 1.49 and 1.92, respectively. It can be seen that, the trend of $M$ exerted on the PMMA and $\mathrm{ZnO}$ nanowires is opposite to that of the silver nanowire, and the $U$ reaches the minimum at $\theta= \pm 90^{\circ}$, which indicate that the PMMA and $\mathrm{ZnO}$ nanowires always experience an optical torque rotating them towards the equilibrium state of $\theta= \pm 90^{\circ}$. As examples,
Figs. $6 \mathrm{~b}$ and $\mathrm{c}$ show the optical field distributions for the PMMA and $\mathrm{ZnO}$ with $\theta=90^{\circ}$ and $s=0$, where the calculated corresponding torque are $M=0$ for both. Further simulation and calculation show that the PMMA and $\mathrm{ZnO}$ nanowires can be oriented by varying the relative distance between the two FPs. Figure S6 in Supplementary Information, as an example, shows the calculated $M$ and $U$ versus $\theta$ at $s=-0.5 \mu \mathrm{m}$ for the $\mathrm{ZnO}$ nanowire. The result indicates that $\theta=75^{\circ}$ is the equilibrium state. Therefore, the method can be used for controllable orientation of dielectric nanowires as well.

To know the three-dimensional trapping ability, trapping force in $x, y$, and $z$ directions near the equilibrium state at $s=0$ was studied (see Fig. S7). The results show that the two counter-propagating laser beams provide a restoring force to trap the nanowire at equilibrium state where the force equals zero. Additionally, it should be also noted that although the irradiation of the laser beam will induce an optical thermal effect on the surface of silver nanowire ${ }^{21,22}$, using heat transfer theory, the thermal effect can be ignored in this experiment (see Fig. S8).

Generally, optical orientation is realized using polarization force. It has been used to orient several kinds of nanowires ${ }^{6,16,23}$. For carbon nanotube (outer diameter $110-170 \mathrm{~nm}$, length $5 \mu \mathrm{m}$ ) and microtubule (diameter $25 \mathrm{~nm}$, length $8 \mu \mathrm{m}$ ) oriented by photonic crystal tweezers $^{23}$, the estimated stiffness coefficients are $2 \times 10^{-20}$ and 9 $\times 10^{-20} \mathrm{~J} / \mathrm{rad}^{2} \cdot \mathrm{mW}$, respectively. For silver nanowires oriented by conventional optical tweezers or analogue ${ }^{6,16}$, the stiffness coefficients were demonstrated to gradually decrease with the increasing length of the nanowires. Especially, for silver nanowire with $6.5 \mu \mathrm{m}$ length and $100 \mathrm{~nm}$ diameter, the estimated stiffness coefficient is $6 \times$ $10^{-20} \mathrm{~J} / \mathrm{rad}^{2} \cdot \mathrm{mW}$ (Ref. 16). In our experiment, the measured stiffness coefficient for silver nanowire $(6.5 \mu \mathrm{m}$ length, $600 \mathrm{~nm}$ diameter $)$ is as high as $\sim 2 \times 10^{-19} \mathrm{~J} / \mathrm{rad}^{2} \cdot \mathrm{mW}$. Moreover, it is expected to be further increased by decreasing the distance $d$ to enhance the radiation field exerted on the silver nanowire. Therefore, we believe that the method can also be used to stably orient thinner or shorter nanowires.

In conclusion, a strategy for realizing precise orientation of single silver nanowire using two fiber probes is proposed and demonstrated. With a laser of $980 \mathrm{~nm}$ wavelength launched into the two fiber probes, the nanowire, which is suspended in water and trapped between the two probes, was rotated and oriented flexibly by varying the relative distance between the two fiber probes with the stability of orientation on the order of $10^{-19} \mathrm{~J} / \mathrm{rad}^{2} \cdot \mathrm{mW}$. Owing to the advantages of handy and easy to set up without complicated components and process, the flexible method for precise orienting nanowires could find potential applications in different assemblies to "pickand-place" one-dimentional nanostructures.

\section{Methods}

Fabrication of FPs. The FPs were fabricated by drawing commercial single-mode optical fibers (connector type: FC/PC; core diameter: $9 \mu \mathrm{m}$; cladding diameter: $125 \mu \mathrm{m}$; Corning Inc.) by a flame-heating technique. At first, the polymer jacket of the fiber was stripped off with a fiber stripper to obtain a bare fiber of 50-mm in length and $125-\mu \mathrm{m}$ in diameter. After that, the bare fiber was kept heating for about $1 \mathrm{~min}$ with a heating region of about $4 \sim 5 \mathrm{~mm}$ to reach its melting point. Then, with a drawing speed of about $0.5 \mathrm{~mm} / \mathrm{s}$ applied on the heating region, the fiber gradually tapered off with its diameter decreasing from 125 to $8 \mu \mathrm{m}$ with a length of about $1.6 \mathrm{~mm}$. Finally, increasing the drawing speed to about $4 \mathrm{~mm} / \mathrm{s}$ until the fiber was broken with an abrupt tapered tip (see insets I and III of Fig. 1a).

Description of experimental setup. The experimental setup is depicted in Fig. 1a. A computer interfaced optical microscope (Union, Hisomet II) with a charge coupled device (CCD, Sony iCY-SHOT, DXC-S500) camera was used for observing and capturing experimental images. The specifications of the objective, including magnification, numerical aperture (NA) and working distance (WD) are $\times 100$ (NA $=0.73, \mathrm{WD}=1.0 \mathrm{~mm})$. Fiber probe $1(\mathrm{FP} 1)$ and fiber probe $2(\mathrm{FP} 2)$ were fixed by tunable six-axis microstages (Kohzu Precision Co., Ltd., $50 \mathrm{~nm}$ in resolution) in an opposite direction. The two tips of the FPs were immersed in the water suspension in which the silver nanowires are dispersed. The silver nanowire suspension is dripped on a glass slide that is placed on a 2D translation stage (resolution: $50 \mathrm{~nm}$ ). A $980 \mathrm{~nm}$ laser (LU0980M330, Lumics, Germany) was used as the light source because the 


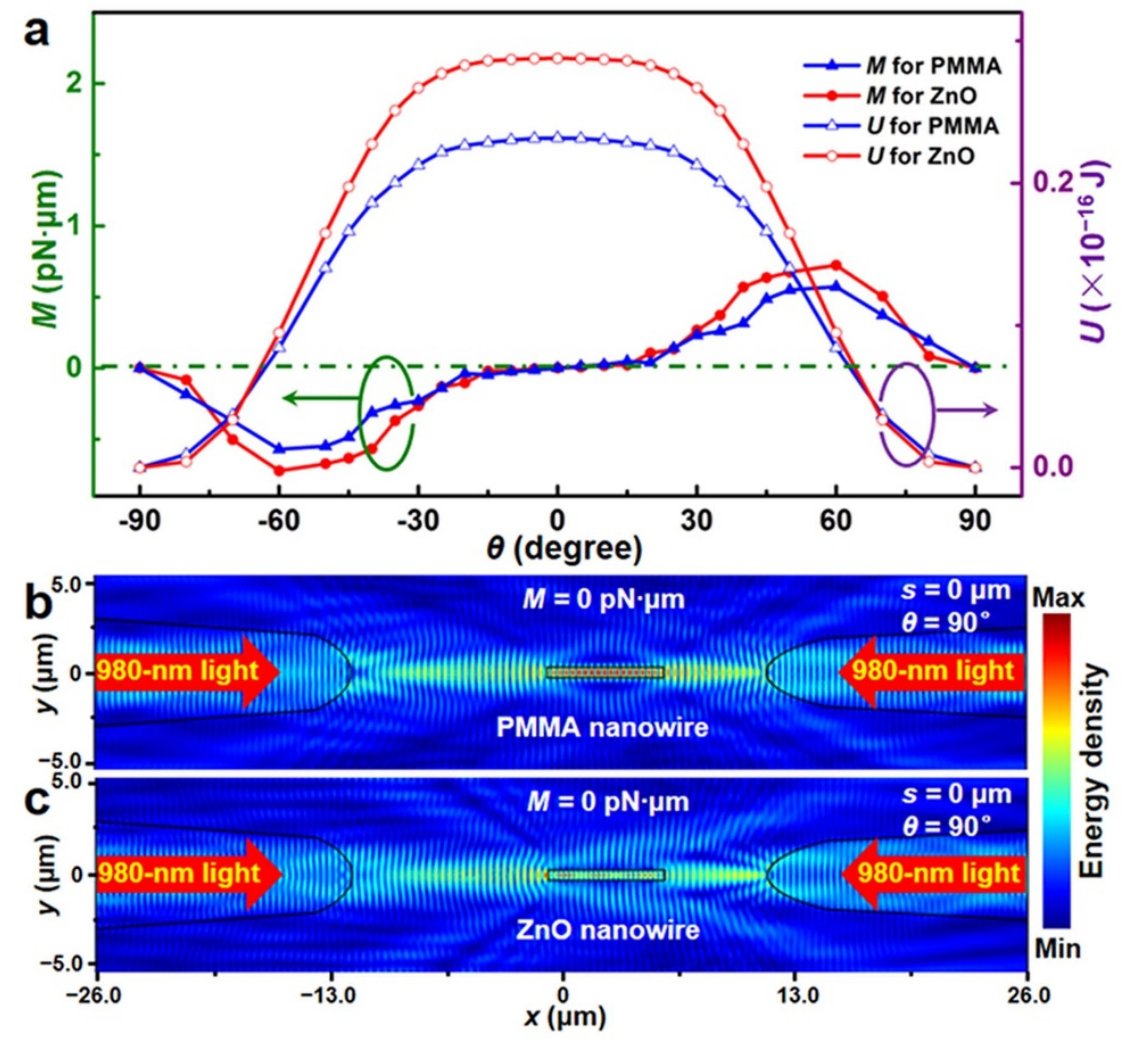

Figure 6 Caculated optical torque $M$, corresponding rotational potential $U$, and simulated optical field distribution for PMMA and ZnO nanowires. (a) $M$ and $U$ versus $\theta$ for PMMA (blue line) and $\mathrm{ZnO}$ (red line) nanowires (6.5- $\mu \mathrm{m}$ length and 600-nm diameter). (b) Simulated time averaged energy density distribution for the PMMA nanowire. (c) Simulated time averaged energy density distribution for the $\mathrm{ZnO}$ nanowire.

$980 \mathrm{~nm}$ laser exhibits relatively low water absorptivity which can avoid obviously thermal effect. The laser was coupled into the FP1 and FP2 by a Y-branch coupler (1:1 splitting ratio).

Preparation of silver nanowire suspension. The synthesized nanowires were diluted in deionized water with the assistance of ultra-sonication for 5 mins to get silver nanowire dispersed suspension. The weight ratio of nanowires to water is about $1: 1,000$. Then a droplet $(\sim 5 \mu \mathrm{L})$ of suspension was injected on a clean glass slide with a micro-syringe.

1. Ditlbacher, H. et al. Silver nanowires as surface plasmon resonators. Phys. Rev. Lett. 95, 257403 (2005).

2. Halas, N. J., Lal, S., Chang, W., Link, S. \& Nordlander, P. Plasmons in strongly coupled metallic nanostructures. Chem. Rev. 111, 3913-3961 (2011).

3. Yan, R., Gargas, D. \& Yang, P. Nanowire photonics. Nature Photon. 3, 569-576 (2008).

4. Fang, Y. et al. Branched silver nanowires as controllable plasmon routers. Nano Lett. 10, 1950-1954 (2010).

5. Novotny, L. \& Hulst, N. V. Antennas for light. Nature Photon. 5, 83-90 (2011).

6. Tong, L., Miljković, V. D. \& Käll, M. Alignment, rotation, and spinning of single plasmonic nanoparticles and nanowires using polarization dependent optical forces. Nano Lett. 10, 268-273 (2010).

7. Dorfmüller, J. et al. Plasmonic nanowire antennas: experiment, simulation, and theory. Nano Lett. 10, 3596-3603 (2010).

8. Jones, P. H. et al. Rotation detection in light-driven nanorotors. ACS Nano 3, 3077-3084 (2009).

9. Maragò, O. M., Jones, P. H., Gucciardi, P. G., Volpe, G. \& Ferrari, A. C. Optical trapping and manipulation of nanostructures. Nature Nanotech 8, 807-819 (2013).

10. Pauzauskie, P. J. et al. Optical trapping and integration of semiconductor nanowire assemblies in water. Nature Mater. 5, 97-101 (2006).

11. Zhong, M., Wei, X., Zhou, J., Wang, Z. \& Li, Y. Trapping red blood cells in living animals using optical tweezers. Nature Commun. 4, 1768 (2013).

12. Padgett, M. \& Bowman, R. Tweezers with a twist. Nature Photon. 5, 343-348 (2011).

13. Dholakia, K. \& Čižmár, T. Shaping the future of manipulation. Nature Photon. 5, 335-342 (2011).

14. Neves, A. A. R. et al. Rotational dynamics of optically trapped nanofibers. Opt. Express 18, 822-830 (2010).
15. Padgett, M., Courtial, J. \& Allen, L. Light's orbital angular momentum. Phys. Today 57, 35-40 (2004).

16. Yan, Z. et al. Three-dimensional optical trapping and manipulation of single silver nanowires. Nano Lett. 12, 5155-5161 (2012).

17. Xin, H., Xu, R. \& Li, B. Optical trapping, driving, and arrangement of particles using a tapered fibre probe. Sci. Rep. 2, 818 (2012).

18. Sun, Y. \& Xia, Y. Large-scale synthesis of uniform silver nanowires through a soft, self-seeding, polyol process. Adv. Mater. 14, 833-837 (2002).

19. Johnson, P. B. \& Christy, R. W. Optical constants of the noble metals. Phys. Rev. B 6, 4370-4379 (1972).

20. Borghese, F., Denti, P., Saija, R. \& Iati, M. A. Radiation torque on nonspherical particles in the transition matrix formalism. Opt. Express 14, 9508-9521 (2006)

21. Baffou, G., Quidant, R. \& García de Abajo, F. J. Nanoscale control of optical heating in complex plasmonic systems. ACS Nano 4, 709-716 (2010).

22. Wang, K., Schonbrun, E., Steinvurzel, P. \& Crozier, K. B. Trapping and rotating nanoparticles using a plasmonic nano-tweezer with an integrated heat sink. Nature Commun. 2, 469 (2011)

23. Kang, P., Serey, X., Chen, Y. \&Erickson D . Angular orientation of nanorods using nanophotonic tweezers. Nano Lett. 12, 6400-6407 (2012).

\section{Acknowledgments}

This work was supported by the National Natural Science Foundation of China (Nos. 11274395 and 61205165), the Guangdong Natural Science Foundation (No.

S2013010012187), and the Program for Changjiang Scholars and Innovative Research Team in University (IRT13042).

\section{Author contributions}

B.L. supervised the project; X.X. and H.X. performed the simulation and calculation; X.X., H.L. and C.C. performed the experiments; X.X., H.L. and B.L. discussed the results and wrote the manuscript.

\section{Additional information}

Supplementary information accompanies this paper at http://www.nature.com/ scientificreports

Competing financial interests: The authors declare no competing financial interests. 
How to cite this article: Xu, X.H., Cheng, C., Xin, H.B., Lei, H.X. \& Li, B.J. Controllable orientation of single silver nanowire using two fiber probes. Sci. Rep. 4, 3989; DOI:10.1038/ srep03989 (2014) (c) (i) (-) $\Theta$ This work is licensed under a Creative Commons AttributionNonCommercial-NoDerivs 3.0 Unported license. To view a copy of this license, visit http://creativecommons.org/licenses/by-nc-nd/3.0 